\title{
A time-saving and cheap strategy to prepare large mesoporous materials for efficient $\mathrm{CO}_{2}$ adsorption
}

Qiqi Zhang 1,2, Guojie Zhang 1, 2, *, Huangyu Yan ${ }^{1,2}$, Yongfa zhang 1,2, Jun Liu ${ }^{3,4}$, Haizhu Cheng 1,2

${ }^{1}$ State Key Laboratory of Clean and Efficient Coal Utilization, Taiyuan University of Technology, Taiyuan 030024, Shanxi, China

${ }^{2}$ Key Laboratory of Coal Science and Technology, Ministry of Education, Taiyuan University of Technology, Taiyuan 030024, Shanxi, China

3 College of Chemistry and Chemical Engineering, Taiyuan University of Technology, Taiyuan 030024, Shanxi, China

${ }^{4}$ National Engineering Laboratory for Multi Flue Gas Pollution Control Technology and Equipment, School of Environment, Tsinghua University, Beijing 100084, China

* Corresponding author's E-mail: zhgjdoc@126.com; zhangguojie@tyut.edu.cn

\section{Supporting Information}

Calculations of $\mathrm{CO}_{2}$ adsorption capacity and amine efficiency

Figure S1 LS sample loaded with $80 \%$ TEPA.

Figure S2 Stability of the adsorbent after steam treatment. 


\section{Calculations of $\mathrm{CO}_{2}$ adsorption capacity and amine efficiency}

The adsorption capacity of $\mathrm{CO}_{2}$ is described by Eq. (1)

$$
q_{a}=\frac{1}{w} \times\left[\int_{0}^{t} Q \times \frac{C_{0}-C}{1-C} \times d t\right] \times \frac{T_{0}}{T} \times \frac{1}{V_{m}}
$$

where $q_{a}$ is the adsorption amount of $\mathrm{CO}_{2}(\mathrm{mmol} / \mathrm{g}) ; w$ is the weight of sorbent $(\mathrm{g}) ; C_{0}$ and $C$ are the $\mathrm{CO}_{2}$ content at the inlet and outlet of reactor; $t$ is the adsorption time (s); $Q$ is the mixed gas intake $(\mathrm{mL} / \mathrm{min}) ; T$ is the $\mathrm{CO}_{2}$ adsorption temperature $(\mathrm{K}), T_{0}$ is $273 \mathrm{~K}$, and $V_{m}$ is $22.4 \mathrm{~mL} / \mathrm{min}$. When $C$ is equal to $C_{0}$, the $\mathrm{CO}_{2}$ capture capacity is called the saturated capture capacity $\left(q_{s}\right)$.

Amine efficiency is calculated by Eq. (2) as follows.

$$
\mathrm{Ea}=\frac{q_{e}}{n_{N}}
$$

where $E a$ represents the amine efficiency $\left(\mathrm{mmol} \mathrm{CO}_{2} / \mathrm{mmol} \mathrm{N}\right), q_{e}$ is saturated capture capacity $(\mathrm{mmol} / \mathrm{g}), n_{N}$ represents the molar content of the $\mathrm{N}$ element in the adsorbent (mmol/g).

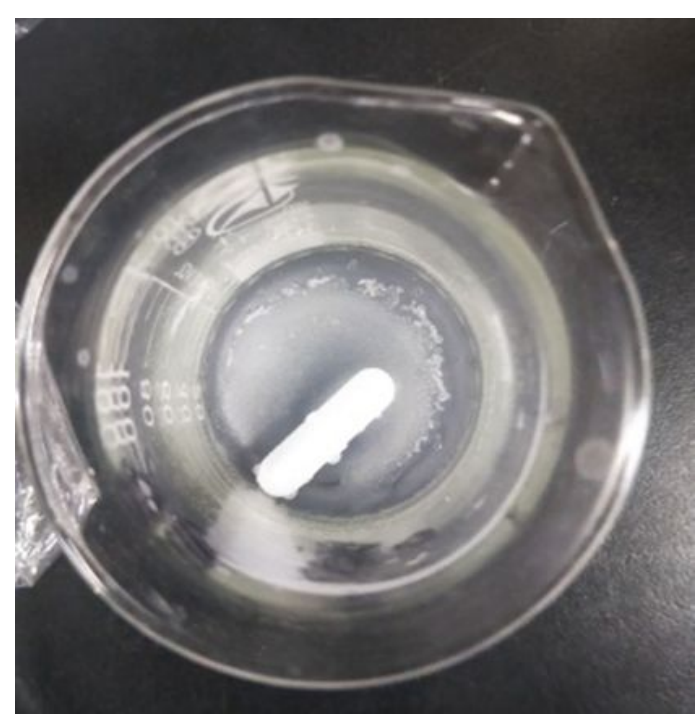

Fig. S1 LS sample loaded with $80 \%$ TEPA 


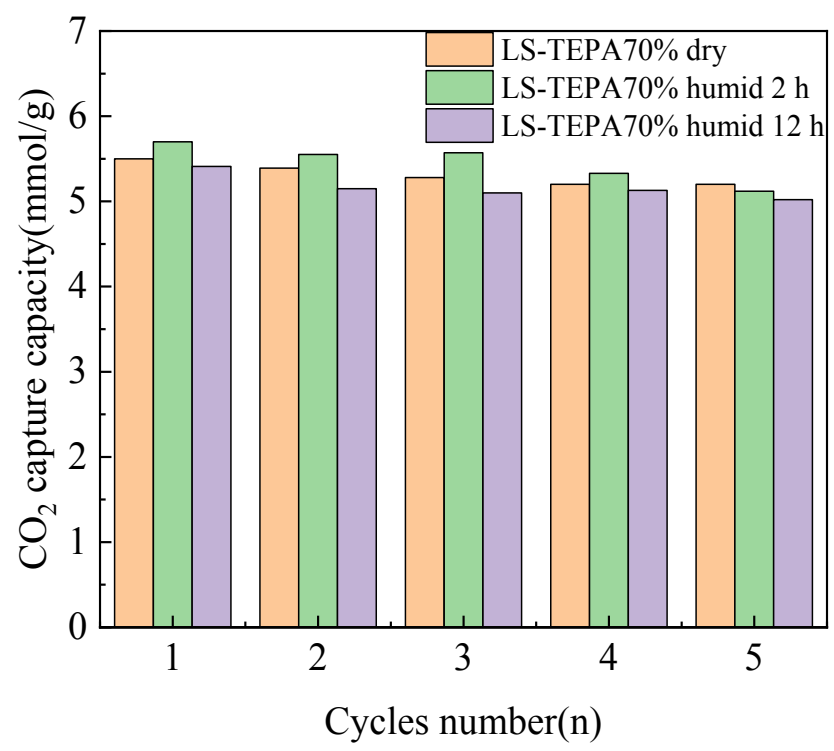

Fig. S2 Stability of the adsorbent after steam treatment 